Marquette University
e-Publications@Marquette

Civil and Environmental Engineering Faculty Research and Publications

$9-15-2018$

\title{
Properties and Mechanisms of Self-Sensing Carbon Nanofibers/ Epoxy Composites for Structural Health Monitoring
}

\author{
Yanlei Wang \\ Dalian University of Technology \\ Yongshuai Wang \\ Dalian University of Technology \\ Baolin Wan \\ Marquette University, baolin.wan@marquette.edu \\ Baoguo Han \\ Dalian University of Technology \\ Gaochuang Cai \\ University of Luxembourg
}

See next page for additional authors

Follow this and additional works at: https://epublications.marquette.edu/civengin_fac

Part of the Civil Engineering Commons

\section{Recommended Citation}

Wang, Yanlei; Wang, Yongshuai; Wan, Baolin; Han, Baoguo; Cai, Gaochuang; and Li, Zhizheng, "Properties and Mechanisms of Self-Sensing Carbon Nanofibers/Epoxy Composites for Structural Health Monitoring" (2018). Civil and Environmental Engineering Faculty Research and Publications. 218.

https://epublications.marquette.edu/civengin_fac/218 


\section{Authors}

Yanlei Wang, Yongshuai Wang, Baolin Wan, Baoguo Han, Gaochuang Cai, and Zhizheng Li

This article is available at e-Publications@Marquette: https://epublications.marquette.edu/civengin_fac/218 
Marquette University

e-Publications@Marquette

\section{Civil and Environmental Engineering Faculty Research and Publications/College of Engineering}

This paper is NOT THE PUBLISHED VERSION; but the author's final, peer-reviewed manuscript. The published version may be accessed by following the link in th citation below.

Composite Structures, Vol. 200 (September 15, 2018): 669-678. DOI. This article is (C) Elsevier and permission has been granted for this version to appear in e-Publications@Marquette. Elsevier does not grant permission for this article to be further copied/distributed or hosted elsewhere without the express permission from Elsevier.

\section{Properties and Mechanisms of Self-Sensing Carbon Nanofibers/Epoxy Composites for Structural Health Monitoring}

\section{Yanlei Wang}

State Key Laboratory of Coastal and Offshore Engineering, School of Civil Engineering, Dalian University of Technology, Dalian, Liaoning 116024, China

Yongshuai Wang

State Key Laboratory of Coastal and Offshore Engineering, School of Civil Engineering, Dalian University of Technology, Dalian, Liaoning 116024, China

Baolin Wan Department of Civil, Construction and Environmental Engineering, Marquette University, Milwaukee, WI

Baoguo Han

State Key Laboratory of Coastal and Offshore Engineering, School of Civil Engineering, Dalian University of Technology, Dalian, Liaoning 116024, China

Gaochuang Cai

Laboratory of Solid Structures, University of Luxembourg, Luxembourg L1359, Luxembourg 


\title{
Zhizheng Li
}

\section{State Key Laboratory of Coastal and Offshore Engineering, School of Civil Engineering, Dalian University of Technology, Dalian, Liaoning 116024, China}

\begin{abstract}
In this paper, carbon nanofibers (CNFs) with high aspect ratio were dispersed into epoxy matrix via mechanical stirring and ultrasonic treatment to fabricate self-sensing CNFs/epoxy composites. The mechanical, electrical and piezoresistive properties of the nanocomposites filled with different contents of CNFs were investigated. Based on the tunneling conduction and percolation conduction theories, the mechanisms of piezoresistive property of the nanocomposites were also explored. The experimental results show that adding CNFs can effectively enhance the compressive strengths and elastic moduli of the composites. The percolation threshold of the CNFs/epoxy composites is 0.186 vol\% according to the modified General Effective Media Equation. Moreover, the stable and sensitive piezoresistive response of CNFs/epoxy composites was observed under monotonic and cyclic loadings. It can be demonstrated that adding CNFs into epoxy-based composites provides an innovative means of self-sensing, and the high sensitivity and stable piezoresistivity endow the CNFs/epoxy composites with considerable potentials as efficient compressive strain sensors for structural health monitoring of civil infrastructures.
\end{abstract}

\section{Keywords}

Carbon nanofibers (CNFs), Piezoresistivity, Nanocomposites, Mechanical properties, Electrical properties

\section{Introduction}

Due to fatigue loads, environmental factors and a range of natural disasters, civil infrastructures often suffer deterioration, damage accumulation or even sudden collapse. Therefore, structural health monitoring (SHM) is widely used to evaluate and monitor the performance of infrastructures. SHM is normally performed by measuring real-time strain values of key positions in the structure, which provides a diagnosis of the current status to determine if it is necessary to repair the structure [1]. However, the conventional strain sensors, which are made of thin metal foils or semiconductors, normally have poor durability and limited sensitivity (their gauge factor is usually about 2). Therefore, various strain sensors with high durability and sensitivity

(e.g., acoustic sensors, magnetic sensors, and piezoresistive sensors) for SHM have been developed [2], [3], [4], [5].

As one of them, using piezoresistive composites to fabricate strain sensors has attracted extensively attention over the last decades due to their simple structures, excellent durability, and high sensitivity [3], [5], [6], [7]. The piezoresistive composites refer to a combination of dielectric or semiconducting matrix (e.g. rubber [8], epoxy [3], [9], [10], [11], [12], [13], [14], and cement [15], [16], [17], [18]) and electrically conductive reinforcement (e.g. carbon nanotubes [9], [12], [15], carbon nanofibers [3], [19], [20], graphene nanoplatelets [13], carbon black [10], [11], and nickel powder [21]). These composites are generally based on the use of conductive fillers, which create an electrical network whose resistance is dependent on the distance between fillers and the piezoresistivity of the fillers themselves [22].

Advances in nanotechnology have enabled the synthesis of "smart" materials which could ultimately replace the function presently carried out by instrumentations. Carbon nanomaterials, such as carbon nanotubes (CNTs) and carbon nanofibers (CNFs), have attracted considerable attention due to their outstanding potentials to improve the physical and electrical properties of traditional materials, which are emerging as a leading solution for 
fabricating piezoresistive strain sensors [14], [23], [24], [25], [26]. A number of studies have been performed to investigate the applications of carbon nanomaterials [3], [12], [27], [28]. Shen et al. [12] investigated the changes of electrical resistance of the carbon black (CB) and CNTs filled epoxy composites under compression, swelling due to water absorption and temperature variation. It was observed that the electrical resistance decreased under compression, while increased upon swelling due to water absorption for all samples, which indicates that the composites had excellent piezoresistive behaviors. Park et al. investigated the self-sensing of CNFs/epoxy composites with two different nanofiber aspect ratios [3]. They pointed out that the composites containing CNFs with high aspect ratio exhibited better self-sensing than that with lower aspect ratio. In addition, considerable studies reported the application of nanocomposite sensors in the field of electrochemical and biological monitoring [29], [30], [31], [32].

The aforementioned studies indicate that it is feasible to fabricate piezoresistive sensors by using carbon nanomaterials for strain monitoring. However, there were limited studies on the application of piezoresistive sensors to embed in concrete structures for SHM. Meanwhile, more attentions had been focused on the cement-based composites due to their good compatibility to concrete structure. Zhang et al. [17] studied the multifunctionality of cement based composites with electrostatic self-assembled CNTs/nano carbon black composites filler. It was found that the piezoresistive properties of the composites containing 2.40 vol\% filler were stable and sensitive, and the maximum relative resistivity change was $25.4 \%$ when the stress amplitude was $10 \mathrm{MPa}$. However, both the compressive strengths and elastic moduli of the composites decreased with the increase of the concentration of CNFs. Xiao et al. [5] investigated the selfmonitoring properties of concrete columns with embedded cement-based strain sensors. The comparison between the monitored results of cement-based sensors and those of traditional displacement transducers indicated that the cement-based sensors had nice strain-sensing abilities [5]. Nevertheless, cementbased sensors are greatly affected by environmental humidity. Therefore, humidity insulation method should be used to guarantee the sensing precision of cement-based composites under various ambient conditions [5]. Additionally, the polarization effect of cement matrix adversely affects the stability of the monitoring [33]. Moreover, the monitoring range of the cement-based compressive strain sensor is limited, which is normally smaller than $0.5 \%$ [34]. The maximum compressive strains of steel tube or fiber reinforced polymers (FRP) confined concrete members are typically in the range of $1.5 \%$ to $3.0 \%$. Therefore, the current cementbased compressive strain sensors are not able to monitor these structural members when they are subjected to large deformation or tested to failure.

Epoxy has excellent chemical resistance, wear resistance, electric insulation, waterproof function and large deformation range. If it is used as the matrix for the compressive strain sensors, it could not only eliminate the effects of humidity and polarization, but also enable the whole process monitoring of confined concrete structures subjected to compression. Compared to multiwall and single-wall carbon nanotubes, CNFs have relatively low cost, which is a favorable factor in practical engineering applications [20]. Therefore, it is worth to study the behaviors of epoxy-based composites containing CNFs to explore the possibility of using such nanocomposites as piezoresistive sensors for structural health monitoring.

This research systematically studied the effects of the contents of CNFs on the mechanical, electrical and piezoresistive properties of CNFs/epoxy composites. The mechanical properties of the nanocomposites fabricated with different contents of CNFs were studied firstly. Secondly, the electrical properties of the CNFs/epoxy composites without loading were investigated and theoretical percolation threshold was confirmed based on the General Effective Media Equation. Thirdly, different loading schemes including monotonic, constant amplitude cyclic and incremental amplitude cyclic loadings were applied to the nanocomposites to examine the stability and sensitivity of their piezoresistive responses. Finally, the 
mechanisms of piezoresistive behavior of the nanocomposites were explored based on tunneling conduction and percolation conduction theories.

\section{Experimental program}

\subsection{Materials}

The epoxy used as the matrix of the nanocomposites was produced by Tianjin Swancor Wind Power Materials Co., Ltd., China. The epoxy is mixed by two parts: SWANCOR 2511-1A and SWANCOR 2511-1BS with a ratio of 10:3 in weight, or 30:11 in volume. It has low viscosity, moderate gel time, nice mechanical properties, high heat deflection temperature (HDT), and good wettability to carbon fibers. The carbon nanofibers (Pyrograf-III, grade PR-24-XT-HHT) with a diameter of 70-200 $\mathrm{nm}$ and a length of 50-200 $\mu \mathrm{m}$ were supplied by Pyrograf Products, Inc., USA. The CNFs in the amount of $0.2 \%, 0.5 \%, 1.0 \%, 1.5 \%, 2.0 \%$, and $3.0 \%$ by weight of epoxy (i.e., $0.116 \%$, $0.29 \%, 0.58 \%, 0.87 \%, 1.16 \%$, and $1.74 \%$ by volume of composite, respectively) were used in this research. The corresponding mixture types are called 0.116 vol\%, 0.29 vol\%, 0.58 vol\%, 0.87 vol\%, 1.16 vol\%, and 1.74 vol\% mixtures, respectively, in this paper. Acetone was used as diluting agent in the amount of $2 \%$ by volume of composite. Copper wire mesh with size of $20 \mathrm{~mm} \times 30 \mathrm{~mm}$ was used as the electrodes.

\subsection{Specimens}

It is well established that the homogeneity of nanofibers dispersion into the polymer matrix is one of the most important factors affecting the nanocomposite's electrical and mechanical performance. Several dispersion methods were recommended in literature to avoid agglomeration and to obtain high percolation in the composites [3], [12], [35], [36]. In this paper, two methods, including mechanical stirring and ultrasonic treatment, were used to disperse CNFs. In order to facilitate the dispersion of CNFs, drying of CNFs was carried out by putting them in oven at $60^{\circ} \mathrm{C}$ for $5 \mathrm{~min}$ to remove the moisture. The CNFs/epoxy composites were prepared by following procedure (Fig. 1): (1) All materials were weighed according to the designed mixture ratio. (2) Different amounts of CNFs ( 0.116 vol\%, 0.29 vol\%, 0.58 vol\%, 0.87 vol\%, 1.16 vol\%, and 1.74 vol\%) were dispersed into acetone by using a mechanical stirrer (Model SFJ-400, Shanghai Modern Environmental Engineering Technology Co., Ltd., China) at high speed (1500 r/min) for $10 \mathrm{~min}$, and then sonicated by Branson 2800 Ultrasonic Cleaner (Model 2510 E-DTH, 100 W 40KHz, Branson Ultrasonic Co., Ltd., USA) for $8 \mathrm{~h}$ at $20^{\circ} \mathrm{C}$ to get CNFs-acetone mixture. (3) Heated (at $60^{\circ} \mathrm{C}$ for $2 \mathrm{~min}$ ) SWANCOR 2511-1A was dissolved in CNFs-acetone mixture via stirring at high speed for $20 \mathrm{~min}$ and ultrasonically dispersing at $60^{\circ} \mathrm{C}$ for $8 \mathrm{~h}$ to get a slurry-like mixture. (4) The mixture was placed in a vacuum oven (Model DZ-2BC, Tianjin Taisite Instrument Co., Ltd., China) to remove acetone and air bubbles. (5) After the mixture was cooled, the curing agent (SWANCOR 2511-1BS) was added and mixed by mechanical stirring at low speed $(500 \mathrm{r} / \mathrm{min}$ ) for $5 \mathrm{~min}$. (6) The CNFs/epoxy mixture was poured into a silicone mold $(20 \mathrm{~mm} \times 20 \mathrm{~mm} \times 40 \mathrm{~mm}$ ), which was brushed a layer of oil for easily remove the specimen after curing, and two copper wire mesh electrodes were embedded in the mixture (Fig. 2). No copper mesh electrodes were added to the specimens for testing the mechanical properties. (7) The specimens were pre-cured at room temperature for $24 \mathrm{~h}$ followed by a post-cure for an additional $8 \mathrm{~h}$ at $80^{\circ} \mathrm{C}$.

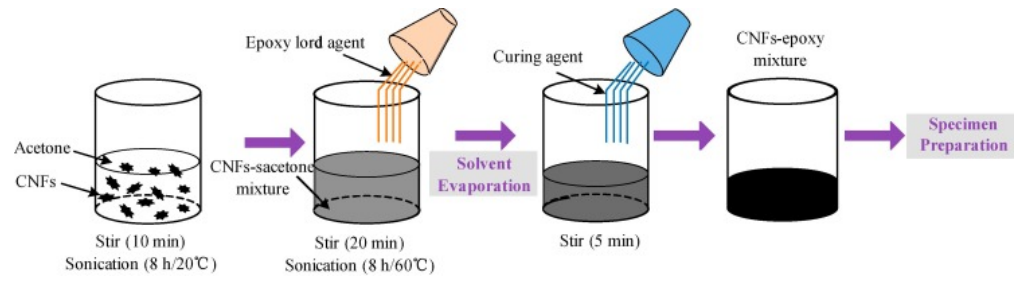

Fig. 1. Preparation process of the nanocomposites. 


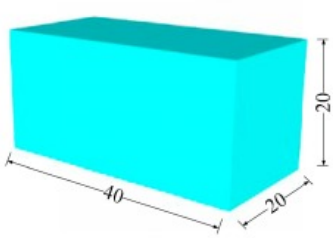

(a)

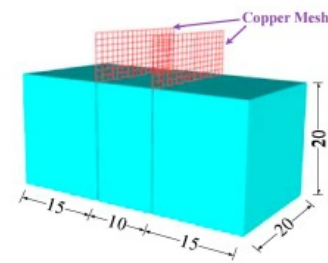

(b)

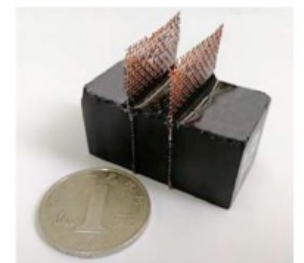

(c)

Fig. 2. Schematic and picture of specimens: (a) schematic of specimens for test of mechanical properties; (b) Schematic of specimens for test of piezoresistive properties; (c) picture of a specimen for test of piezoresistive properties. (Units in $\mathrm{mm}$ ).

\subsection{Measurements}

\subsubsection{Mechanical measurement}

For each type of CNFs/epoxy composites, three identical specimens were prepared and tested. The compression test was performed by using an electronic universal testing machine (Model WDE-200E, Jinan Gold Testing Machines Inc., China) at room temperature. A displacement control protocol was used in the compression testing with a constant loading rate of $0.4 \mathrm{~mm} / \mathrm{min}$. Two pairs of strain gauges were glued symmetrically at the opposite sides of the middle section of the specimen to test strains. In each pair of the strain gauges, one was in the axial direction and the other was in the lateral direction. The DH3820 strain acquisition device (Donghua Testing Technology Co., Ltd., China) was employed to record strain data. The compressive strengths, Poisson's ratios and elastic moduli of the CNFs/epoxy composites were calculated from the uniaxial compression test results.

\subsubsection{Electrical measurement}

The electrical resistance of the specimens without loading was tested by two-electrode method using a Keithley 2100 digital multimeter (Keithley Instruments Inc., USA). The two copper wire meshes were served as the electrical contacts for conducting the current and measuring the voltage, simultaneously. The two-electrode method was chosen to measure the electrical resistance rather than the four-electrode method due to the better suitability, in terms of implementation [37]. The volumetric electrical resistivity (the resistance per unit volume in the bulk material) $\rho$ is determined by:

(1) $\rho=R \frac{A}{L}$

where $R$ is the measured electrical resistance, $A$ is the cross-sectional area of the sample, and $L$ is the separation between the two electrodes.

\subsubsection{Piezoresistive measurement}

The specimens with 0.29 vol\% of CNFs were chosen to test piezoresistivity under different loading rates from $0.2 \mathrm{~mm} / \mathrm{min}$ to $5 \mathrm{~mm} / \mathrm{min}$ to study the effect of loading rate on the piezoresistive properties. Subsequently, the specimens with 0.29 vol\%, 0.58 vol\% and 1.16 vol\% of CNFs, respectively, were subjected to the monotonic, constant amplitude cyclic and incremental amplitude cyclic loadings. For the constant amplitude cyclic loading, the peak value was $25 \mathrm{MPa}$ and the valley value was $0.75 \mathrm{MPa}$ (shown in Fig. $3 \mathrm{a}$ ) so that the specimen was kept in the approximate elastic state. The incremental amplitude cyclic loading was achieved by gradually increasing the loading step in steps of $10 \mathrm{MPa}, 15 \mathrm{MPa}, 20 \mathrm{MPa}, 25 \mathrm{MPa}$ and $35 \mathrm{MPa}$, respectively, and each step was cycled three times (shown in Fig. 3b). For each type of CNFs/epoxy composites, six identical specimens were prepared. Three specimens were tested under monotonic compressive loads until their failures, and the other three specimens were first subjected to constant cyclic loading and then subjected to incremental 
amplitude cyclic loading to simulate realistic loading conditions. During the test process, the DC resistance was tested by two-electrode method via the Keithley 2100 digital multimeter and the axial strain was monitored by one pair of strain gauges which were glued symmetrically at the opposite sides of the middle section of the specimen. The experimental setup was shown in Fig. 4.
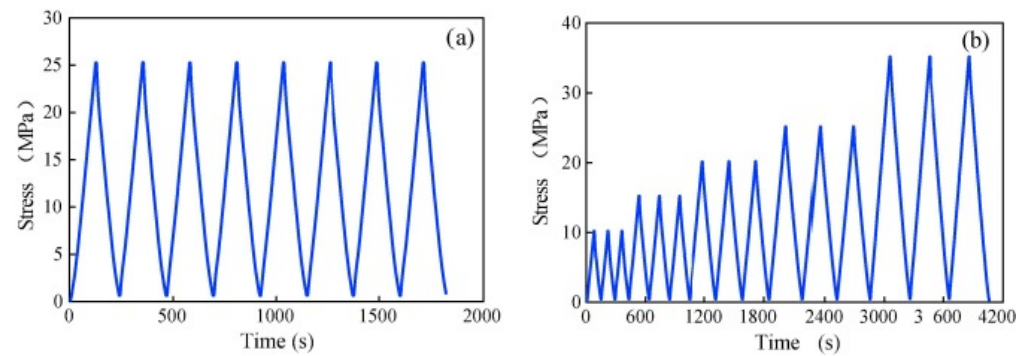

Fig. 3. Loading history: (a) constant amplitude under cyclic loading; (b) incremental amplitude under cyclic loading.

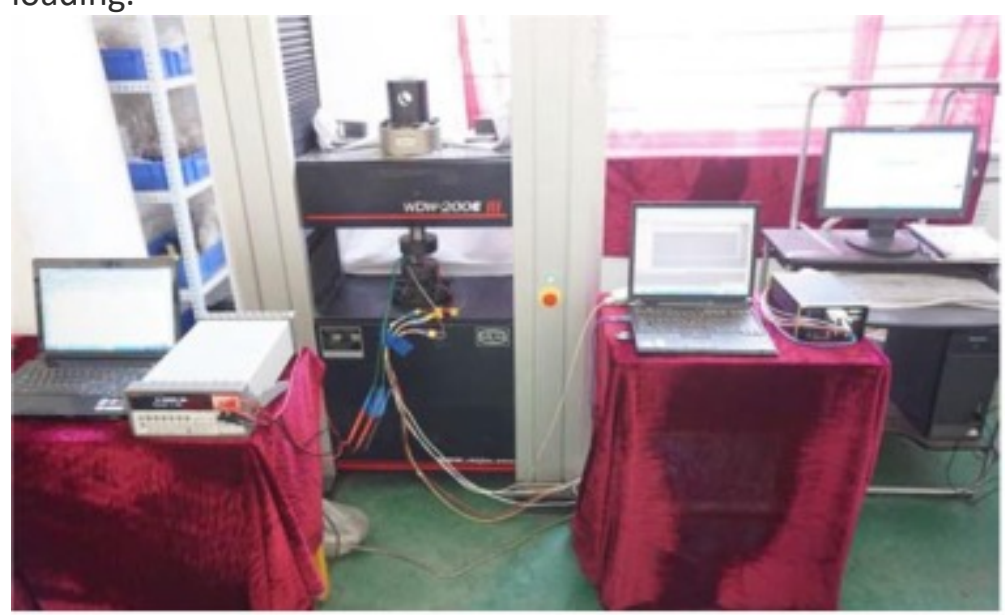

Fig. 4. Experimental setup of piezoresistive test.

\subsubsection{Microstructure characterization}

The morphologies of the CNFs/epoxy composites were examined by using a scanning electron microscope (SEM) (Nova NANOSEM450, FEI Inc., USA). The samples used for optical microscopic analysis were cut from the failure regions of the fractured specimens. Before the SEM observation, the fractured surfaces were gold coated using an ion sputter equipment to improve the conductance for proper investigation. There are many factors affecting the CNF dispersion in the epoxy matrix. In order to reduce the variation among SEM images, five samples were investigated by the SEM for each group of specimens with same CNF content.

\section{Results and discussions}

\subsection{Microstructure}

It should be noted that the samples with the same content of CNFs had the similar microscopic characters at the fracture surface and all SEM images shown in this study are the typical results. Fig. 5 shows the SEM pictures of nanocomposites filled with different amount of CNFs ( 0 vol\%, 0.29 vol\%, 0.58 vol\% and 1.16 vol\%).

Comparison of the nine images in Fig. 5 indicates that CNFs were dispersed uniformly in the epoxy matrix when the contents of CNFs were not more than 0.58 vol\% (Fig. $5 \mathrm{~b}-\mathrm{e}$ ), while a little more agglomerations of CNFs were observed when the content of CNFs was 1.16 vol\% (Fig. $5 \mathrm{f}-\mathrm{i}$ ). However, most of the CNFs can be dispersed in the epoxy matrix to form conductive networks throughout the structure. The achievement of the good dispersion of CNFs is significant for enhancing the nanocomposites' properties, especially the mechanical properties and the piezoresistive reproducibility. 

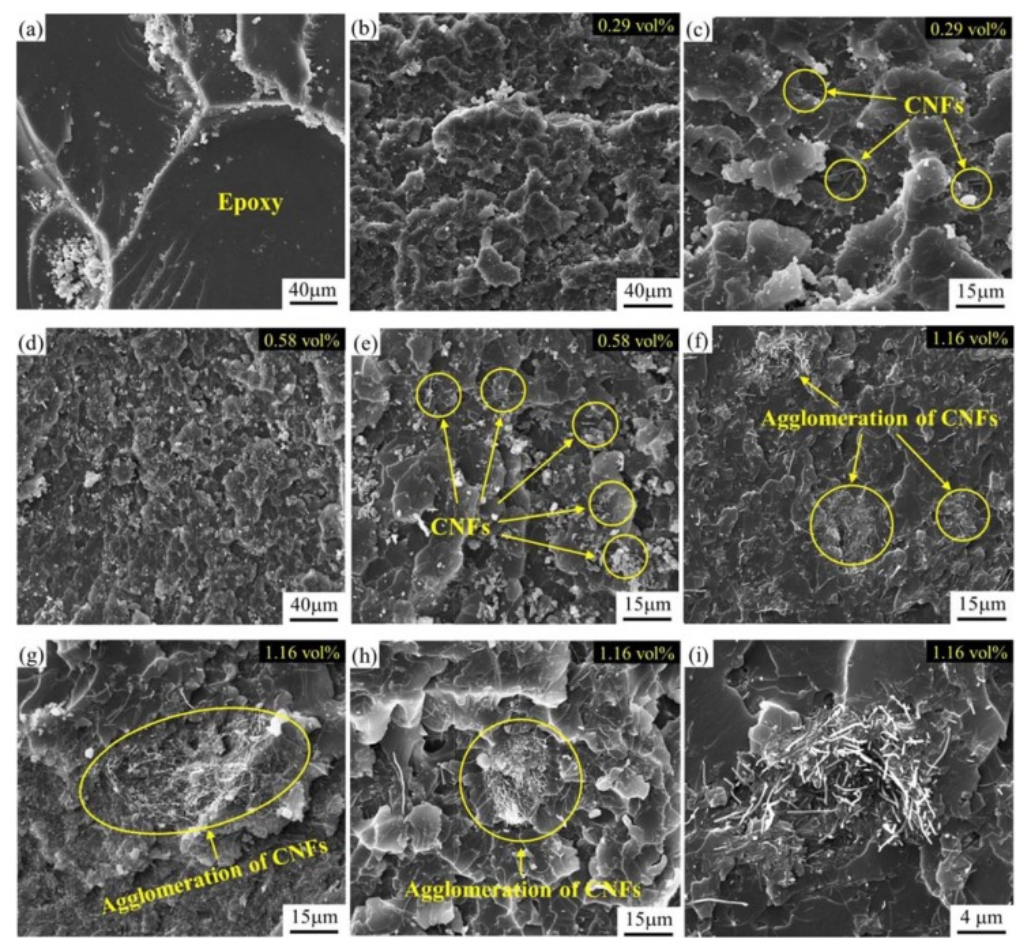

Fig. 5. SEM images of the nanocomposites filled with (a) 0 vol\%; (b) and (c) 0.29 vol\%; (d) and (e) 0.58 vol\%; (f) to (i) 1.16 vol\% of CNFs.

\subsection{Mechanical properties}

Mechanical properties including compressive strength, elastic modulus and Poisson's ratio, were determined for the CNFs/epoxy composites from the uniaxial compressive tests. Fig. 6a shows the static compression stressstrain curves for the composites containing different contents of CNFs. The changes in compressive strength, elastic modulus and Poisson's ratio versus the CNFs content are shown in Fig. $6 \mathrm{~b}-\mathrm{d}$, respectively. The column charts show the average values with error bars obtained from three samples for each type of composite. As shown in Fig. 6a, the composites with various contents of CNFs exhibited a similar stress-strain relationship. The stress-strain curves show a nearly linear relationship before the stress reached around 30-40 MPa, while the nonlinear responses were observed under larger stress. Fig. $6 \mathrm{~b}$ indicates that strong influence of the introduction of the CNFs on the compressive strength. The compressive strength increased significantly when the content of CNFs was increased up to 0.58 vol\% and then decreased slightly when the content of CNFs further increased to 1.16 vol\%. The compressive strengths of the composites filled with 0.58 vol\% CNFs reached the maximum of $77.6 \mathrm{MPa}$, which was $16 \%$ higher than that of the control specimens. As shown in Fig. $6 \mathrm{c}$ and $d$, the elastic moduli and the Poisson's ratios of the nanocomposites varied by changing the contents of the CNFs. The elastic modulus increased, while the Poisson's ratio decreased with the increase of the content of CNFs. However, CNFs had a greater impact on the elastic moduli of the composites, which increased $26 \%$ for the specimens with 1.16 vol\% compared to the control specimens. 

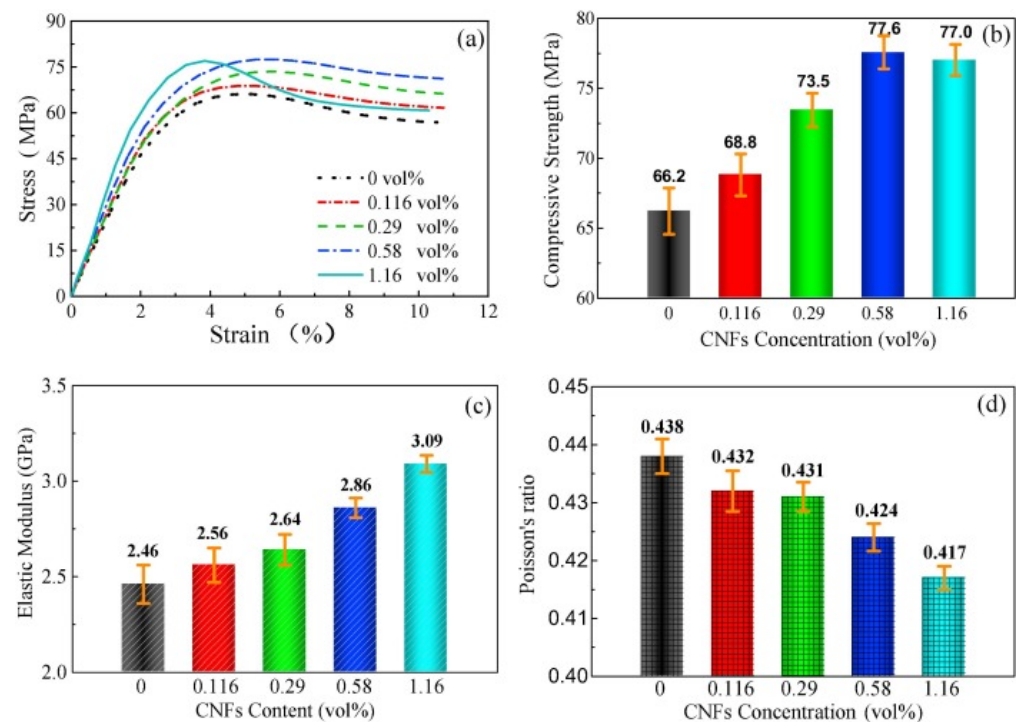

Fig. 6. Mechanical properties of CNFs/epoxy composites: (a) stress-strain relationship for CNFs/epoxy composites; (b) compressive strengths of CNFs/epoxy composites; (c) elastic moduli of CNFs/epoxy composites; (d) Poisson's ratio of CNFs/epoxy composites.

The interfacial bond between the CNFs and the epoxy matrix enables the load to be transferred to the CNFs through the interface, which is an important factor for enhancing the mechanical properties of the composite. CNFs have larger strengths and elastic moduli than those of epoxy matrix. Because part of the load is supported by the CNFs and the lateral deformation is reduced due to the constraint of the CNFs, the compressive strengths and elastic moduli of the nanocomposites are improved. Meanwhile, the pulling effect of the CNFs in the epoxy matrix also constrained the transverse deformation of the composites, resulting in a decrease in the Poisson's ratio of the composite compared to the control epoxy composite. However, when the content of CNFs was increased to 1.16 vol\%, the inhomogeneous dispersion of CNFs into the matrix leaded to the appearance of much agglomerations (Fig. $5 \mathrm{f}-\mathrm{i}$ ). The agglomeration of the CNFs causes defects in the composites, resulting in a decrease in the strengths of the composites. This is the reason why a decrease in the compressive strengths of composites filled with 1.16 vol\% CNFs happened as shown in Fig. 6 b.

\subsection{Electrical properties}

Fig. 7 shows the measured electrical resistivity of the CNFs/epoxy composites versus the contents of CNFs. It is observed that a significant decrease of resistivity when the content of CNFs increased from 0 to 0.58 vol\%, and then a much smaller decrease rate at higher content of CNFs. The content range where the resistivity varied drastically is called the percolation threshold. Therefore, the possible percolation threshold zone of the CNFs/epoxy composites ranges from 0.116 vol\% to 0.58 vol\% (the corresponding resistivity dropped from $1.01 \times 10^{8} \Omega \cdot \mathrm{cm}$ to $2.64 \times 10^{4} \Omega \cdot \mathrm{cm}$ ) in this study. The electrical resistivity of the CNFs/epoxy composites reached the minimum value of $2.93 \times 10^{3} \Omega \cdot \mathrm{cm}$ when the CNFs content was $1.74 \mathrm{vol} \%$. In order to confirm the percolation threshold of CNFs in the nanocomposites from theoretical investigations, a statistical model described by the General Effective Media (GEM) equation was explored. It takes into account the conductivities of constituent materials and is given by [38]:

(2) $\frac{(1-\varphi)\left(\rho_{e}^{-1 / t}-\rho^{-1 / t}\right)}{\rho_{e}^{-1 / t}+A \rho^{-1 / t}}+\frac{\varphi\left(\rho_{c}^{-1 / t}-\rho^{-1 / t}\right)}{\rho_{c}^{-1 / t}+A \rho^{-1 / t}}=0$

(3) $A=\left(1-\varphi_{c}\right) / \varphi_{c}$ 
where $\varphi$ is the volume fraction of CNFs; $\rho, \rho_{e}$, and $\rho_{c}$ are the electrical resistivity of the nanocomposite, epoxy, and CNFs, respectively; $\varphi_{c}$ is the critical volume fraction of CNFs (i.e., the percolation threshold, and when $\varphi$ is larger than $\varphi_{c}$, CNFs can form conductive paths in the nanocomposite); and $t$ is a shape exponent. The electrical resistivity of epoxy used in this study (about $10^{8}-10^{10} \Omega \cdot \mathrm{cm}$ ) is much larger than that of CNFs $\left(10^{-1}-10^{-3} \Omega \cdot \mathrm{cm}\right.$ ). Therefore, the resistivity of epoxy can be assumed to approach infinite [39], i.e., $\rho_{e} \rightarrow \infty$, and a simplified solution of Eq. (2) can be obtained as:

(4) $\rho \approx k\left(\varphi-\varphi_{c}\right)^{-t} \operatorname{for} \varphi \geqslant \varphi_{c}$

where $k$ is a constant. The best fit of our experimental resistivity data to log-log plots of Eq. (4) (shown in the inset of Fig. 7) gives the values of $\varphi_{c}$ and $t$ for CNFs/epoxy composites are 0.186 vol\% and 1.59 , respectively.

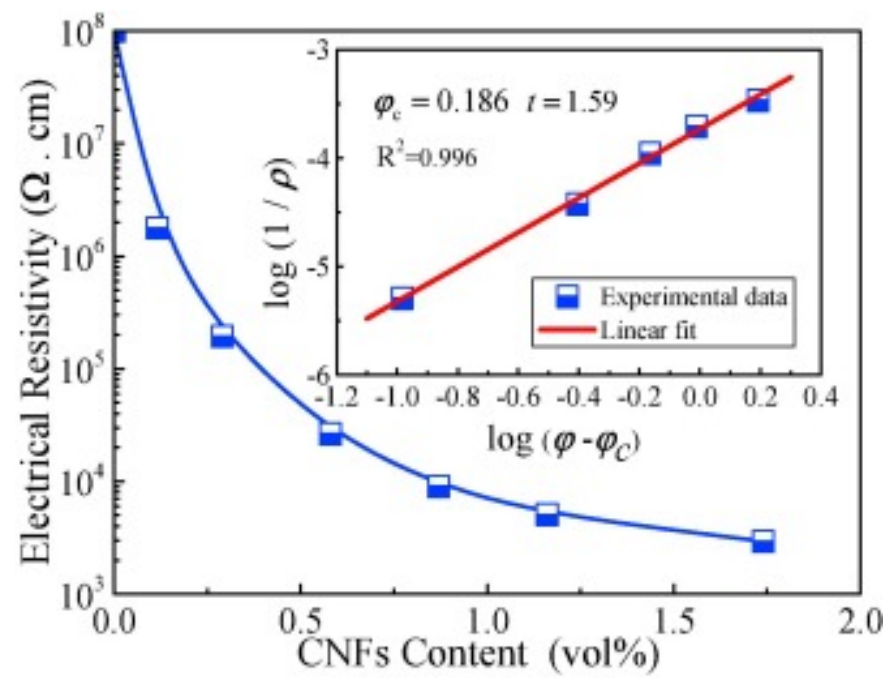

Fig. 7. Electrical resistivity of CNFs/epoxy composites as a function of CNFs content. (Inset: logarithm of conductivity as a function of logarithm of reduced volume fraction and fit to a line).

The results agree with the paths theory, i.e. the number of conduction paths between the CNFs increases in the composite with the increase of the CNFs content. Therefore, more contacts between the CNFs might be the major reason causing the decrease of the electrical resistivity of the composites. Meanwhile, when the CNFs content is increased, the gaps between the CNFs become narrower and the electronic charges can transport between CNFs easier, which also causes smaller resistivity. A sparse conductive network is formed in the composites with low content of CNFs. Therefore, they have a lower electrical conductivity compared with those with more CNFs.

\subsection{Piezoresistive properties}

\subsubsection{Effect of loading rates on piezoresistive properties}

The major purpose of this research is to study the possibility of using the CNFs/epoxy composites as a sensor for structural health monitoring of civil infrastructures. Therefore, the piezoresistive properties of the composites were investigated and are discussed in this section.

Since the content of 0.29 vol\% is close to the percolation threshold of the CNFs/epoxy composites, the specimens with 0.29 vol\% CNFs were chosen to investigate the effect of loading rate on their piezoresistive properties and to select the appropriate loading rate for the subsequent experimental tests. Five loading rates $(0.2,0.4,0.8,1.5$ and $5.0 \mathrm{~mm} / \mathrm{min}$ ) were applied to load specimens. Fig. 8 shows the variation of stress and relative resistivity as the functions of strain, respectively, under different loading rates. 


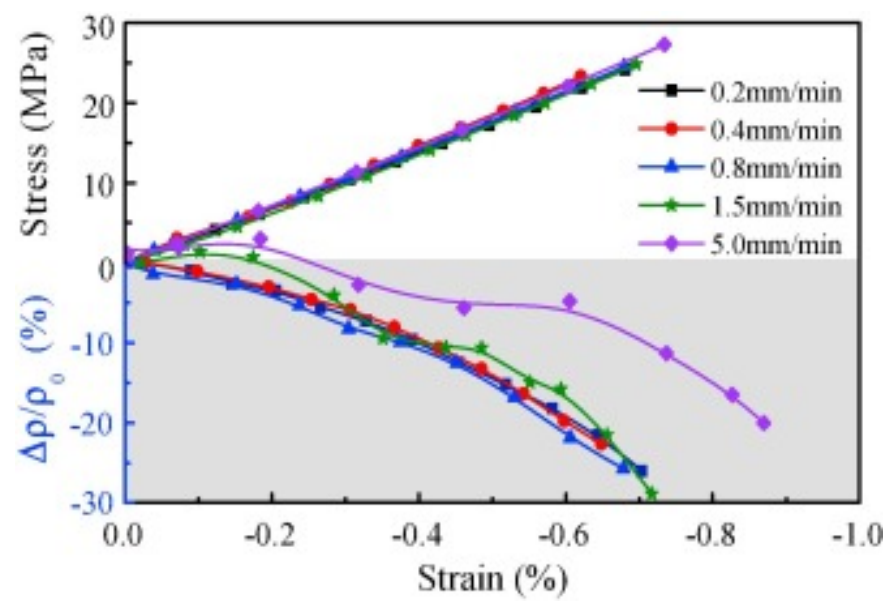

Fig. 8. Responses of stress and relative resistivity to strain under compression with different loading rates.

The stress-strain curves plotted in Fig. 8 indicate that the deformation of the composite during test was in the elastic region, which agrees well with the results in Section 3.2, i.e., the stress-strain curves presented an approximate linear relationship before the stress reached $30 \mathrm{MPa}$. Fig. 8 shows that the piezoresistive properties of the composites were almost unaffected by the loading rate lower than $0.4 \mathrm{~mm} / \mathrm{min}$, while the fluctuation of the curve of the relative change in electrical resistivity was observed under larger loading rate. Especially, the curve shows a noticeable fluctuation when the loading rate exceeds $1.5 \mathrm{~mm} / \mathrm{min}$, which reveals that large loading rates will deteriorate the stability of the piezoresistive properties of the composites. The conductive networks formed by CNFs inside the specimens vary uniformly as the slow deformation of the specimens under small loading rates, leading to the stable behavior of the piezoresistive properties. When the loading rate is large, the inhomogeneous deformation in the longitudinal direction of the specimen leads to the sudden change of the internal conductive network, which causes the resistance of the composites unstable during the loading process. Therefore, in the following tests, the loading rate of $0.4 \mathrm{~mm} / \mathrm{min}$ was chosen to ensure the composites possessing stable piezoresistive properties.

\subsubsection{Piezoresistive properties under monotonic loading}

To evaluate the piezoresistive properties of the CNFs/epoxy composites, the samples were tested under monotonic compressive loading till failure. Fig. 9 shows the typical variation in relative resistivity under monotonic loading for the CNFs/epoxy composites containing 0.29 vol\%, 0.58 vol\% and 1.16 vol\% of CNFs.

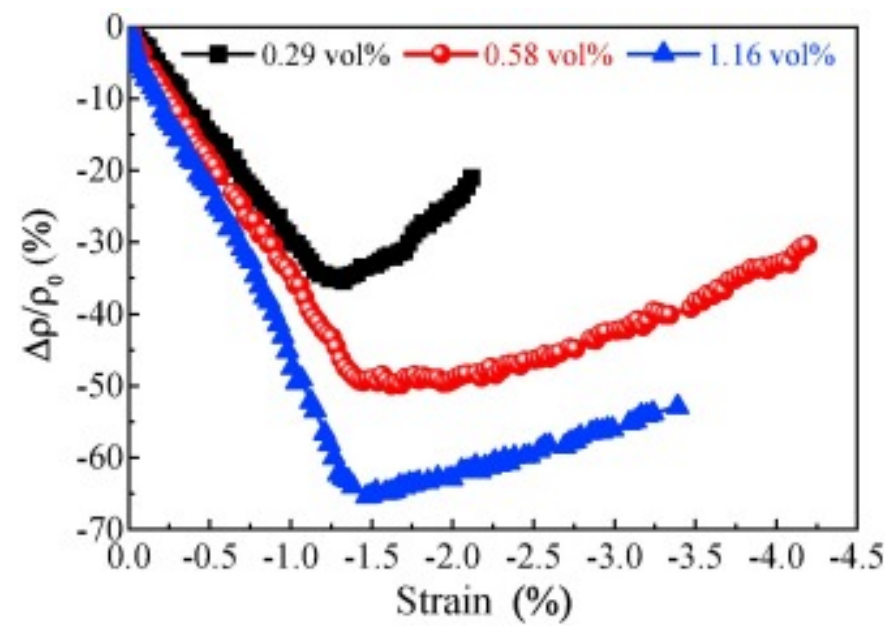

Fig. 9. Response of relative resistivity to compressive strain under monotonic static loading. 
It can be observed from Fig. 9 that the composites filled with CNFs exhibit strong piezoresistive behaviors when they are subjected to uniaxial compression. Regardless of the content of CNFs, the relative change in electrical resistivity, $\Delta \rho / \rho_{0}$, increased linearly with the increase of the applied strain up to a certain value, and then decreased thereafter. The maximum relative resistivity changes are $37 \%, 50 \%$ and $68 \%$, for the nanocomposites filled with 0.29 vol\%, 0.58 vol\%, and 1.16 vol\% of CNFs, respectively.

Compared with the stress vs strain curves (Fig. 6a), the transition points in Fig. 9 are close to the elastic deformation limits of the specimens, beyond which the specimens enter the plastic zone. The compression of the epoxy matrix under load leads to a reduction of the distances between the neighboring CNFs. According to the tunneling conduction theory, a reduction of tunneling gap provokes an evident decrease of electrical resistivity [39]. When the specimen enters plastic stage with the increase of the load, micro-cracks are initiated and developed in the specimens. These micro-cracks generate gaps and increase the distance between CNFs, therefore hinder the tunneling effect. Meanwhile, as the cracks expand and develop with the increase of the load, fewer contacts between CNFs lead to the decrease of conduction paths. These may be the main reasons that the CNFs/epoxy composites exhibited the piezoresistive responses shown in Fig. 9.

The strain sensitivity coefficient $(S)$, also called Gauge Factor, of a strain sensor, which is defined as the ratio of the relative change in electrical resistance $\left.\Delta R / R_{0}\right)$ and the strain $(\varepsilon)$, is given by Eq. (6) [40].

(5) $S=\frac{\Delta R / R_{0}}{\varepsilon}$

where $\Delta R$ is the electrical resistance increment, $R_{0}$ is the initial electrical resistance without loading. The values of strain sensitivity coefficient obtained by linear regression analysis of the data according to Eq. (5) are 29, 37 and 56, respectively. It should be noted that the data used for this analysis is from the elastic region of the nanocomposites. It is widely recognized that the gauge factor of nanocomposite strain sensors is affected by the number of conductive pathways [3], [12], which can be altered by changing the content of CNFs in the nanocomposite. Upon increasing the content of CNFs from 0.29 vol\% to 1.16 vol\%, the strain sensitivity coefficient increases continuously from 29 to 56, which is much higher than that of the traditional electronic resistance strain gauges (about 2).

\subsubsection{Piezoresistive properties under constant amplitude cyclic loading}

In order to simulate realistic loading conditions, piezoresistive behaviors of CNFs/epoxy composites under constant and incremental amplitude cyclic loadings were investigated in this research. To evaluate the piezoresistive stability of CNFs/epoxy composites strain sensors, they were subjected to constant amplitude cyclic loading and unloading (i.e., eight compressing and releasing cycles) with a loading rate of $0.4 \mathrm{~mm} / \mathrm{min}$. Pre-pressure treatment for each specimen was carried out by applying cyclic preconditioning strain before cyclic loading test in order to achieve a stable conductive state.

Fig. 10 shows the typical value of $\Delta \rho / \rho_{0}$ as a function of the number of cycles of loading to $25 \mathrm{MPa}$ and unloading to $0.75 \mathrm{MPa}$ applied to the composites filled with different contents of CNFs. It is observed from Fig. 10 that the instantaneous response of the relative change in resistivity closely follows the change of the strain/stress, which indicates that the resistivity of the specimens varies synchronously with the applied strain/stress. The value of the relative change in resistivity steadily increases with the increase of compression and then gradually decreases during unloading. In the compressing-releasing cycles, the composites with different contents of CNFs essentially recover their resistance after releasing, indicating excellent stability and reproducibility of the piezoresistivity. However, piezoresistive responses of the nanocomposites with lower CNF contents exhibit a slight fluctuation during cyclic loading and unloading. The piezoresistive behavior is due to the nanoscale structural change in the percolation and corresponds to a stochastic separation of the conducting pathways because of the increased matrix strain/stress. Electrons can tunnel through a polymer matrix if the 
distance between the adjacent CNFs is less than the tunneling distance (of the order 5-50 $\mathrm{nm}$ ) [41]. Therefore, during the constant amplitude cyclic loading, the reversible approaching/leaving of the conductive fillers causes the resistivity increase/decrease.
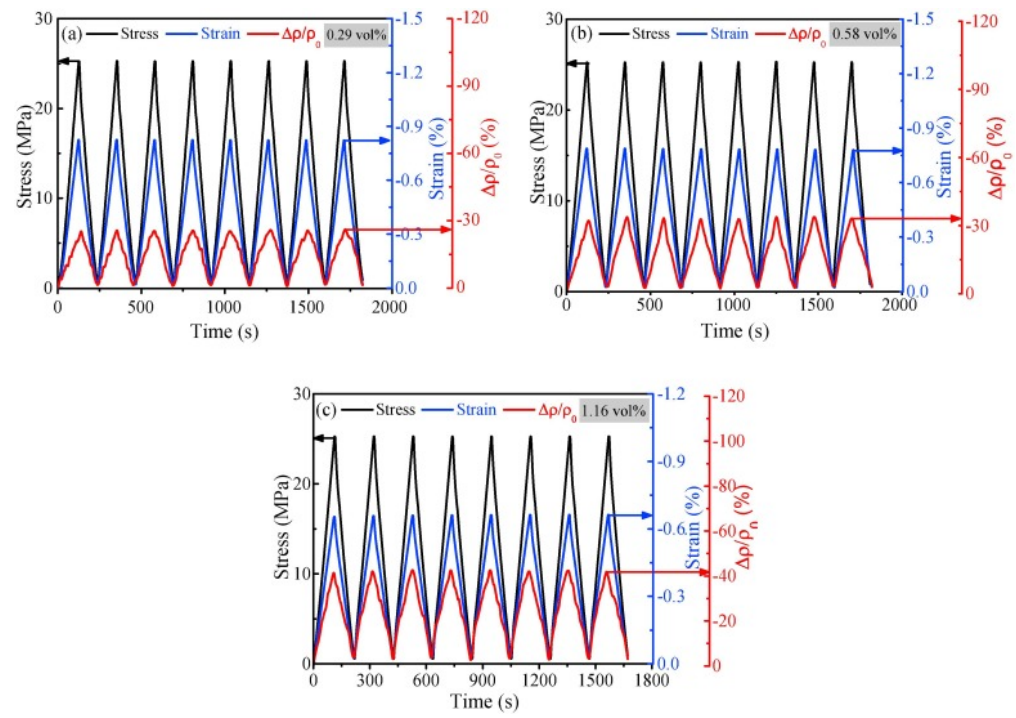

Fig. 10. Time-history relationship between relative change in resistivity and constant cyclic compressive stress/strain for composites filled with (a) 0.29 vol\%; (b) 0.58 vol\%; (c) 1.16 vol\% of CNFs.

\subsubsection{Piezoresistive properties under incremental amplitude cyclic loading}

Composites filled with different contents of CNFs were subjected to incremental amplitude cyclic loading with a loading rate of $0.4 \mathrm{~mm} / \mathrm{min}$. Fig. 11 shows the typical relationships between relative change in resistivity and the strain/stress of the composites filled with different contents of CNFs. The change of resistivity with time has similar trend to that of the incremental amplitude cyclic loading. It can be observed from the figure that the relative change in resistivity has excellent repeatability under cyclic compression regardless of the contents of CNFs in the nanocomposites. The relative change in resistivity of the CNFs/epoxy composites increases with the increase of the cyclic loading amplitude and the increase of the content of CNFs. This phenomenon is due to the increase of CNFs amount and the decrease of the distance between the neighboring CNFs. The distances of the conductive CNFs in the epoxy matrix become smaller with the increase of the loading amplitudes. According to the tunneling conduction theory, a reduction of tunneling gap leads to a significant decrease of the electrical resistivity. Therefore, the larger stress amplitude is applied to the nanocomposite, the more sensitive piezoresistive properties of the composite has. 

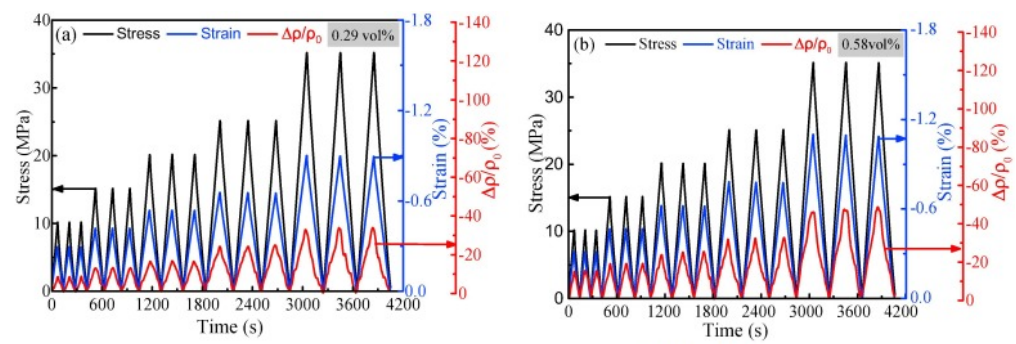

(c)

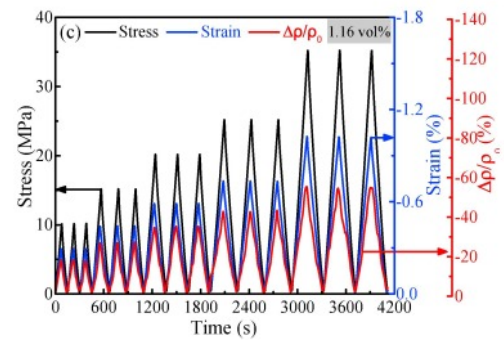

Fig. 11. Time-history relationship between the relative change in resistivity and the incremental cyclic compressive stress/strain for composites filled with (a) 0.29 vol\%; (b) 0.58 vol\%; (c) 1.16 vol\% of CNFs.

\subsection{Mechanisms of piezoresistive properties}

The previous discussions indicate that the piezoresistive properties of the CNFs/epoxy composites are related to the tunneling conduction theory and the percolation conduction theory. When the content of CNFs is relative low (below 0.186 vol\% in this study), tunneling conduction is the major cause for the decreases in the electrical resistivity of the composites. The electrical conductivity is low without loading since there are a few CNFs and no contacts between them as shown in Fig. 12. During loading, the epoxy matrix experiences a much larger deformation in the direction of compression, which results in a reduction of the distance between CNFs (Blue path (1) shown in Fig. 12). Meanwhile, more tunneling paths will be formed (red path (2) shown in Fig. 12). The conductivity of the nanocomposites increases gradually due to the tunneling effects among those neighboring CNFs, although there is no complete conductive path formed by contacting CNFs.

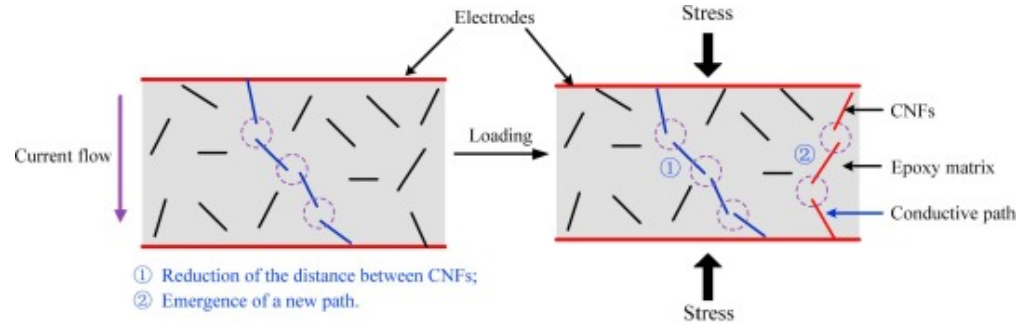

Fig. 12. Schematic diagram of piezoresistive mechanisms of the composites with low contents of CNFs.

When the content of CNFs is relative large (higher than 0.186 vol\%), the electrical conductivity of composites increases remarkably by the percolation conduction as shown in Fig. 13. In this case, the complete electrically conductive paths connect by some CNFs contacting each other (blue paths in Fig. 13), which may be considered as the major cause for the decrease of the electrical resistivity of the composites with large content of CNFs. During compression, more conductive paths (red path shown in Fig. 13) created by the contacts among the CNFs will be formed, which leads to a further decrease in the resistivity of the nanocomposites. During the cyclic loading, the destruction and reconstruction of conductive paths cause the piezoresistive responses of the nanocomposites. 


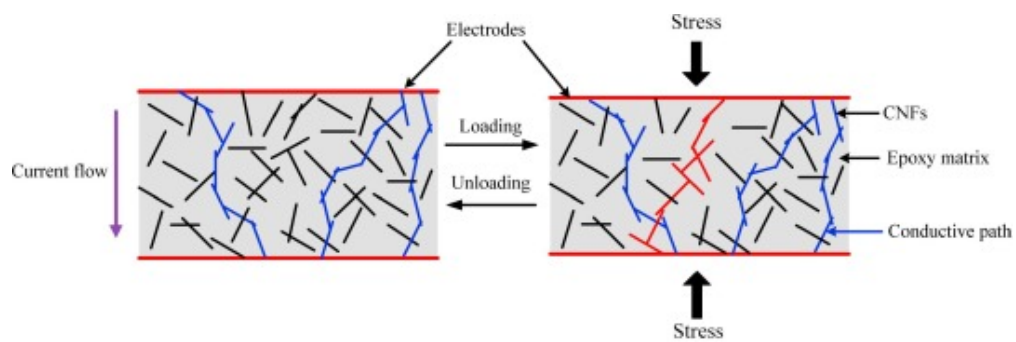

Fig. 13. Schematic diagram of piezoresistive mechanisms of the composites with high contents of CNFs.

\section{Conclusions}

In summary, self-sensing CNFs/epoxy composites were prepared by mechanical stirring and ultrasonic treatment. The mechanical, electrical and piezoresistive properties of the composites filled with different contents of CNFs were investigated. Based on the tunneling conduction and percolation conduction theories, the mechanisms of piezoresistive properties for the nanocomposites were also explored. The following conclusions can be drawn from this study:

(1) SEM results showed that most CNFs can be uniformly dispersed in the epoxy matrix to form the conductive networks. However, a little more agglomerations of CNFs were observed when the CNFs content was relative large, which had adverse effects on the mechanical and piezoresistive properties of the nanocomposites.

(2) The compressive strengths and elastic moduli of the nanocomposites were considerably enhanced by incorporating CNFs. The interfacial bond between the CNFs and the epoxy matrix, facilitating the load to be transferred to the CNFs through the interface, is an important factor for enhancing the mechanical properties of the nanocomposites.

(3) When the content of CNFs was increased, a significant decrease of electrical resistivity of the nanocomposites was followed by a much smaller decrease at higher content of CNFs. The percolation threshold of CNFs in the nanocomposites is 0.186 vol\% via solving the General Effective Media equation.

(4) The CNFs/epoxy composites exhibit a stable and repeatable piezoresistive property under monotonic, constant amplitude cyclic and incremental amplitude cyclic loadings. The gauge factor ranges from 29 to 56 for the nanocomposites filled with CNFs contents from 0.29 vol\% to 1.16 vol\%, which demonstrates that the compressive strain sensors fabricated by these nanocomposites have considerable potentials to be used for structural health monitoring.

\section{Acknowledgments}

This work was supported by the National Key R\&D Program of China (Project No. 2017YFC0703008), the National Natural Science Foundation of China (Project No. 51778102), the Fundamental Research Funds for the Central Universities (Project No. DUT18LK35) and the State Scholarship Fund of China (Project No. 201706060249).

\section{Appendix A. Supplementary data}

Download XML file (252B)Help with xml files

Supplementary data 1. 


\section{References}

[1]S.W.S. Doebling, C.R.C. Farrar, M.B.M. Prime, D.W.D. Shevitz Damage identification and health monitoring of structural and mechanical systems from changes in their vibration characteristics: a literature review, 133, Los Alamos Natl Lab (1996)

[2] M. Shateri, M. Ghaib, D. Svecova, D. Thomson On acoustic emission for damage detection and failure prediction in fiber reinforced polymer rods using pattern recognition analysis Smart Mater Struct, 26 (2017), pp. 1-24

[3] J.M. Park, J.H. Jang, Z.J. Wang, D.J. Kwon, K.L. Devries Self-sensing of carbon fiber/carbon nanofiber-epoxy composites with two different nanofiber aspect ratios investigated by electrical resistance and wettability measurements Compos Part A Appl Sci Manuf, 41 (2010), pp. 1702-1711

[4] J. Zhang, C. Liu, M. Sun, Z. Li An innovative corrosion evaluation technique for reinforced concrete structures using magnetic sensors Constr Build Mater, 135 (2017), pp. 68-75

[5] H. Xiao, H. Li, J. Ou Self-monitoring properties of concrete columns with embedded cement-based strain sensors J Intell Mater Syst Struct, 22 (2011), pp. 191-200

[6] S. Ding, B. Han, X. Dong, X. Yu, Y. Ni, Q. Zheng, et al. Pressure-sensitive behaviors, mechanisms and model of field assisted quantum tunneling composites Polym, 113 (2017), pp. 105-118

[7] R. Liu, H. Xiao, H. Li, L. Sun, Z. Pi, G. Waqar, et al. Effects of nano-SiO ${ }_{2}$ on the permeability-related properties of cement-based composites with different water/cement ratios J Mater Sci, 53 (2018), pp. 4974-4986

[8] H. Aguilar-Bolados, M.A. Lopez-Manchado, J. Brasero, F. Avilés, M. Yazdani-Pedram Effect of the morphology of thermally reduced graphite oxide on the mechanical and electrical properties of natural rubber nanocomposites Compos Part B Eng, 87 (2016), pp. 350-356

[9] H. Xiao, G. Song, H. Li, L. Sun Improved tensile properties of carbon nanotube modified epoxy and its continuous carbon fiber reinforced composites Polym Compos, 36 (2015), pp. 1664-1668

[10] A. Djabali, L. Toubal, R. Zitoune, S. Rechak An experimental investigation of the mechanical behavior and damage of thick laminated carbon/epoxy composite Compos Struct, 184 (2018), pp. 178-190

[11] H.P.S. Abdul Khalil, M. Jawaid, P. Firoozian, M. Amjad, E. Zainudin, M.T. Paridah Tensile, electrical conductivity, and morphological properties of carbon black-filled epoxy composites Int J Polym Anal Charact, 18 (2013), pp. 329-338

[12] J.T. Shen, S.T. Buschhorn, J.T.M. De Hosson, K. Schulte, B. Fiedler Pressure and temperature induced electrical resistance change in nano-carbon/epoxy composites Compos Sci Technol, 115 (2015), pp. 1-8

[13] R. Moriche, M. Sánchez, A. Jiménez-Suárez, S.G. Prolongo, A. Ureña Strain monitoring mechanisms of sensors based on the addition of graphene nanoplatelets into an epoxy matrix Compos Sci Technol, 123 (2016), pp. 65-70

[14] A. Bouhamed, A. Al-Hamry, C. Müller, S. Choura, O. Kanoun Assessing the electrical behaviour of MWCNTs/epoxy nanocomposite for strain sensing Compos Part B Eng, 128 (2017), pp. 91-99

[15] B.S. Sindu, S. Sasmal Properties of carbon nanotube reinforced cement composite synthesized using different types of surfactants Constr Build Mater, 155 (2017), pp. 389-399

[16] B. Han, L. Zhang, C. Zhang, Y. Wang, X. Yu, J. Ou Reinforcement effect and mechanism of carbon fibers to mechanical and electrically conductive properties of cement-based materials Constr Build Mater, 125 (2016), pp. 479-489

[17] L. Zhang, B. Han, J. Ouyang, X. Yu, S. Sun, J. Ou Multifunctionality of cement based composite with electrostatic self-assembled CNT/NCB composite filler Arch Civ Mech Eng, 17 (2017), pp. 354-364

[18] W. Li, X. Li, S.J. Chen, Y.M. Liu, W.H. Duan, S.P. Shah Effects of graphene oxide on early-age hydration and electrical resistivity of Portland cement paste Constr Build Mater, 136 (2017), pp. 506-514 
[19] S. Mondal, L. Nayak, M. Rahaman, A. Aldalbahi, T.K. Chaki, D. Khastgir, et al. An effective strategy to enhance mechanical, electrical, and electromagnetic shielding effectiveness of chlorinated polyethylene-carbon nanofiber nanocomposites Compos Part B Eng, 109 (2017), pp. 155-169

[20] Y. Wang, R. Chang, G. Chen Strain and damage self-sensing properties of carbon nanofibers/carbon fiberreinforced polymer laminates Adv Mech Eng, 9 (2017), pp. 1-11

[21] H. Xiao, M. Liu, G. Wang Anisotropic electrical and abrasion-sensing properties of cement-based composites containing aligned nickel powder Cement Concrete Compos, 87 (2018), pp. 130-136

[22] Alamusi, N. Hu, H. Fukunaga, S. Atobe, Y. Liu, J. Li Piezoresistive strain sensors made from carbon nanotubes based polymer nanocomposites Sensors, 11 (2011), pp. 10691-10723

[23] J. Luo, K.L. Chung, Q. Li, S. Chen, L. Li, D. Hou, et al. Piezoresistive properties of cement composites reinforced by functionalized carbon nanotubes using photo-assisted Fenton Smart Mater Struct, 26 (2017), p. 35025

[24] B.F. Gonçalves, J. Oliveira, P. Costa, V. Correia, P. Martins, G. Botelho, et al. Development of water-based printable piezoresistive sensors for large strain applications Compos Part B Eng, 112 (2017), pp. 344352

[25] H.K. Kim, I.S. Park, H.K. Lee Improved piezoresistive sensitivity and stability of CNT/cement mortar composites with low water-binder ratio Compos Struct, 116 (2014), pp. 713-719

[26] I.W. Nam, S.M. Park, H.K. Lee, L. Zheng Mechanical properties and piezoresistive sensing capabilities of FRP composites incorporating CNT fibers Compos Struct, 178 (2017), pp. 1-8

[27] X. Wei, X. Cao, Y. Wang, G. Zheng, K. Dai, C. Liu, et al. Conductive herringbone structure carbon nanotube/thermoplastic polyurethane porous foam tuned by epoxy for high performance flexible piezoresistive sensor Compos Sci Technol, 149 (2017), pp. 166-177

[28] E. Zegeye, A.K. Ghamsari, Y. Jin, E. Woldesenbet The strain sensing property of carbon nanofiber/glass microballoon epoxy nanocomposite Smart Mater Struct, 22 (2013)

[29] J. Shi, J. Hu, Z. Dai, W. Zhao, P. Liu, L. Zhao, et al. Graphene welded carbon nanotube crossbars for biaxial strain sensors Carbon N Y, 123 (2017), pp. 786-793

[30] E. Arkan, G. Paimard, K. Moradi A novel electrochemical sensor based on electrospun $\mathrm{TiO}_{2}$ nanoparticles/carbon nanofibers for determination of Idarubicin in biological samples J Electroanal Chem, 801 (2017), pp. 480-487

[31] Y. Wang, G. Chen, B. Wan, H. Lin, J. Zhang Behavior of innovative circular ice filled steel tubular stub columns under axial compression Constr Build Mater, 171 (2018), pp. 680-689

[32] M. Adabi, R. Saber, R. Faridi-Majidi, F. Faridbod Performance of electrodes synthesized with polyacrylonitrile-based carbon nanofibers for application in electrochemical sensors and biosensors Mater Sci Eng C, 48 (2015), pp. 673-678

[33] L. Zhang, S. Ding, S. Dong, Z. Li, J. Ouyang, X. Yu, et al. Piezoresistivity, mechanisms and model of cementbased materials with CNT/NCB composite fillers Mater Res Express, 4 (2017), p. 125704

[34] B. Han, J. Ou Embedded piezoresistive cement-based stress/strain sensor Sensors Actuators A Phys, 138 (2007), pp. 294-298

[35] A. Al-Sabagh, E. Taha, U. Kandil, G.A. Nasr, M.M. Reda Taha Monitoring damage propagation in glass fiber composites using carbon nanofibers Nanomaterials, 6 (2016), p. 169

[36] W. Liu, Y. Wang, P. Wang, Y. Li, Q. Jiang, X. Hu, et al. A biomimetic approach to improve the dispersibility, interfacial interactions and toughening effects of carbon nanofibers in epoxy composites Compos Part B Eng, 113 (2017), pp. 197-205

[37] A.O. Monteiro, A. Loredo, P.M.F.J. Costa, M. Oeser, P.B. Cachim A pressure-sensitive carbon black cement composite for traffic monitoring Constr Build Mater, 154 (2017), pp. 1079-1086

[38] D.S. McLachlan Equations for the conductivity of macroscopic mixtures J Phys C Solid State Phys, 19 (1986), pp. 1339-1354 
[39] P. Wang, T. Ding Conductivity and piezoresistivity of conductive carbon black filled polymer composite J Appl Polym Sci, 116 (2010), pp. 2035-2039

[40] S. Luo, W. Obitayo, T. Liu SWCNT-thin-film-enabled fiber sensors for lifelong structural health monitoring of polymeric composites - from manufacturing to utilization to failure Carbon N Y, 76 (2014), pp. 321329

[41] M. Amjadi, Y.J. Yoon, I. Park Ultra-stretchable and skin-mountable strain sensors using carbon nanotubesEcoflex nanocomposites Nanotechnology, 26 (2015) 